\title{
Sets of parts such that the partition function is even
}

\author{
by \\ F. BEN SAÏD (Monastir) and J.-L. Nicolas (Lyon)
}

1. Introduction. $\mathbb{N}_{0}$ and $\mathbb{N}$ denote the set of non-negative integers, resp. positive integers. $\mathcal{A}$ will denote a set of positive integers, and its counting function will be denoted by $A(x)$ :

$$
A(x)=|\{a: a \leq x, a \in \mathcal{A}\}| .
$$

If $\mathcal{A}=\left\{a_{1}, a_{2}, \ldots\right\} \subset \mathbb{N}$ (where $a_{1}<a_{2}<\ldots$ ), then $p(\mathcal{A}, n)$ denotes the number of partitions of $n$ with parts in $\mathcal{A}$, that is, the number of solutions of the equation

$$
a_{1} x_{1}+a_{2} x_{2}+\ldots=n
$$

in non-negative integers $x_{1}, x_{2}, \ldots$ As usual, we shall set

$$
p(\mathcal{A}, 0)=1 \quad \text { and } \quad p(\mathcal{A}, n)=0 \text { for } n<0 .
$$

We shall use the generating function

$$
F(z)=F_{\mathcal{A}}(z)=\sum_{n=0}^{\infty} p(\mathcal{A}, n) z^{n}=\prod_{a \in \mathcal{A}} \frac{1}{1-z^{a}} .
$$

When $\mathcal{A}=\mathbb{N}$ it seems highly probable that the number of integers $n \leq x$ such that $p(\mathbb{N}, n)$ is even is close to $x / 2$ as $x \rightarrow \infty$; but the known results are rather poor (see [7], [9], [10] and the references in them). That is the reason for which, in [7], it was observed that there exist sets $\mathcal{A}$ such that $p(\mathcal{A}, n)$ is even for $n$ large enough. In this paper, we want to investigate the properties of such sets.

For $i=0$ or 1 , if $\mathcal{A} \subset \mathbb{N}$ and there is a number $N$ such that

$$
p(\mathcal{A}, n) \equiv i(\bmod 2) \quad \text { for } n \in \mathbb{N}, n>N,
$$

then $\mathcal{A}$ is said to have property $P_{i}(N)$.

If $i=0$ or $1, \mathcal{B}=\left\{b_{1}, \ldots, b_{k}\right\} \neq \emptyset$ (where $\left.b_{1}<\ldots<b_{k}\right)$ is a finite set of positive integers, $N \in \mathbb{N}$ and $N \geq b_{k}$, then there is (cf. [7]) a unique set

2000 Mathematics Subject Classification: Primary 11P81.

Research partially supported by Franch-Tunisian exchange program, C.M.C.U. no. 99/F 1507 and by CNRS, Institut Girard Desargues, UMR 5028. 
$\mathcal{A} \subset \mathbb{N}$ such that

$$
\mathcal{A} \cap\{1, \ldots, N\}=\mathcal{B}
$$

and having property $P_{i}(N)$; we will denote it by $\mathcal{A}_{i}(\mathcal{B}, N)$.

Let us recall the construction of $\mathcal{A}_{i}(\mathcal{B}, N)$ as described in [7], when, for instance, $i=0$. The set $\mathcal{A}=\mathcal{A}_{0}(\mathcal{B}, N)$ will be defined by recursion. We write $\mathcal{A}_{n}=\mathcal{A} \cap\{1, \ldots, n\}$ so that

$$
\mathcal{A}_{N}=\mathcal{A} \cap\{1, \ldots, N\}=\mathcal{B} .
$$

Assume that $n \geq N+1$ and $\mathcal{A}_{n-1}$ has been defined so that $p(\mathcal{A}, m)$ is even for $N+1 \leq m \leq n-1$. Then set

$$
n \in \mathcal{A} \text { if and only if } p\left(\mathcal{A}_{n-1}, n\right) \text { is odd. }
$$

It follows from the construction that for $n \geq N+1$, we have

$$
p(\mathcal{A}, n)= \begin{cases}1+p\left(\mathcal{A}_{n-1}, n\right) & \text { if } n \in \mathcal{A}, \\ p\left(\mathcal{A}_{n-1}, n\right) & \text { if } n \notin \mathcal{A},\end{cases}
$$

which shows that $p(\mathcal{A}, n)$ is even for $n \geq N+1$. Note that in the same way, any finite set $\mathcal{B}=\left\{b_{1}, \ldots, b_{k}\right\}$ can be extended to a set $\mathcal{A}$ so that $\mathcal{A}_{b_{k}}=\mathcal{B}$ and the parity of $p(\mathcal{A}, n)$ is given for $n \geq N+1$ (where $N$ is any integer such that $N \geq b_{k}$ ).

It will be shown in Proposition 4 that, except in the case $i=1, \mathcal{B}=\{1\}$, the set $\mathcal{A}_{i}(\mathcal{B}, N)$ is always infinite.

By the unicity of the above construction, if the set $\mathcal{A}$ has property $P_{i}(M)$, then, clearly, for any $N \geq M$ and $\mathcal{B}=\mathcal{A} \cap\{1, \ldots, N\}$ we have

$$
\mathcal{A}=\mathcal{A}_{i}(\mathcal{B}, N) \text {. }
$$

If $\mathcal{A} \subset \mathbb{N}$, let $\chi(\mathcal{A}, n)$ denote the characteristic function of $\mathcal{A}$, i.e.,

$$
\chi(\mathcal{A}, n)= \begin{cases}1 & \text { if } n \in \mathcal{A}, \\ 0 & \text { if } n \notin \mathcal{A},\end{cases}
$$

and for $n \geq 1$,

$$
\sigma(\mathcal{A}, n)=\sum_{d \mid n} \chi(\mathcal{A}, d) d=\sum_{d \mid n, d \in \mathcal{A}} d .
$$

It is relevant to consider $\sigma(\mathcal{A}, n)$, since, as shown in [7], taking the logarithmic derivative of $F(z)=F_{\mathcal{A}}(z)$ defined by (1.3) yields

$$
z \frac{F^{\prime}(z)}{F(z)}=\sum_{n=1}^{\infty} \sigma(\mathcal{A}, n) z^{n} .
$$

The main purpose of this paper is to show that for any positive integer $k$ and any set $\mathcal{A}=\mathcal{A}_{i}(\mathcal{B}, N)$, the sequence $\left(\sigma\left(\mathcal{A}, 2^{k} n\right) \bmod 2^{k+1}\right)_{n \geq 1}$ is periodic.

(We denote by $a \bmod b$ the remainder in the Euclidean division of $a$ by $b$.) 
Note that (1.10) has already been proved for $k=0$ in [7], and for $k=1$ in [2]. The result (1.10) will be proved (Theorem 1) in Section 3, and, in the same section, Theorem 2 will specify the smallest period $q_{k}$ of $\sigma\left(\mathcal{A}, 2^{k} n\right) \bmod$ $2^{k+1}$; in particular, $q_{k}$ is always odd. Property (1.10) seems a little surprising; the number 2 appears in it since the question we study is a parity problem.

By the Möbius inversion formula, (1.8) gives

$$
n \chi(\mathcal{A}, n)=\sum_{d \mid n} \mu(d) \sigma(\mathcal{A}, n / d)
$$

where $\mu$ is the Möbius function. If $n$ is odd, by (1.10) with $k=0$, we know the value of $\sigma(\mathcal{A}, n) \bmod 2$, and this allows us to determine $\chi(\mathcal{A}, n)$ from $(1.11)$ for any set $\mathcal{A}=\mathcal{A}_{i}(\mathcal{B}, N)$. This has been done in [8] for $\mathcal{A}=\mathcal{A}_{0}(\{1,2,3\}, 3)$ and in [6] for $\mathcal{A}=\mathcal{A}_{0}(\{1,2,3,4,5\}, 5)$. In [2], the validity of (1.10) for $k=1$ has been used to determine the elements of $\mathcal{A}=\mathcal{A}_{0}(\{1,2,3\}, 3)$ which are congruent to 2 modulo 4 .

Similarly, it is possible to deduce from $(1.10)$ the value of $\chi(\mathcal{A}, n)$ where $n$ is any positive integer. For that, it is convenient for $m$ odd to introduce the sum

$$
S(m, k)=\chi(\mathcal{A}, m)+2 \chi(\mathcal{A}, 2 m)+\ldots+2^{k} \chi\left(\mathcal{A}, 2^{k} m\right) .
$$

If $n=2^{k} m$ with $k \geq 0$ and $m$ odd, (1.8) implies

$$
\sigma(\mathcal{A}, n)=\sigma\left(\mathcal{A}, 2^{k} m\right)=\sum_{d \mid m} d S(d, k)
$$

which, by the Möbius inversion formula, gives

$$
m S(m, k)=\sum_{d \mid m} \mu(d) \sigma(\mathcal{A}, n / d)=\sum_{d \mid \bar{m}} \mu(d) \sigma(\mathcal{A}, n / d),
$$

where $\bar{m}=\prod_{p \mid m} p$ denotes the radical of $m$. In the above sums, $n / d$ is always a multiple of $2^{k}$, so that, from (1.10), the value of $\sigma(\mathcal{A}, n / d)$ and thus the value of $S(m, k)$ are known modulo $2^{k+1}$. Therefore, from (1.12), we can deduce the value of $\chi\left(\mathcal{A}, 2^{i} m\right)$ for $i \leq k$. But, for technical reasons, the calculation can be difficult. We hope to return to this subject in another article.

Finally, in Section 4, we prove in Theorem 3 that, for any $\mathcal{B}$ and $N$, there is a set $\mathcal{B}^{\prime}$ such that $\mathcal{A}_{1}(\mathcal{B}, N)$ and $\mathcal{A}_{0}\left(\mathcal{B}^{\prime}, N+1\right)$ have the same elements with the exception of powers of 2 .

We are pleased to thank K. Belabas and A. Sárközy for several remarks.

2. The Graeffe transformation. Consider the ring of formal power series $\mathbb{C}[[z]]$. For an element

$$
f(z)=a_{0}+a_{1} z+a_{2} z^{2}+\ldots+a_{n} z^{n}+\ldots
$$


of this ring, the product

$$
f(z) f(-z)=b_{0}+b_{1} z^{2}+b_{2} z^{4}+\ldots+b_{n} z^{2 n}+\ldots
$$

is an even power series with

$$
\begin{aligned}
& b_{0}=a_{0}^{2}, \\
& b_{1}=2 a_{0} a_{2}-a_{1}^{2}, \ldots, b_{n}=2\left(\sum_{i=0}^{n-1}(-1)^{i} a_{i} a_{2 n-i}\right)+(-1)^{n} a_{n}^{2} .
\end{aligned}
$$

We shall write $g=\mathcal{G}(f)$ for the series

$$
g(z)=\mathcal{G}(f)(z)=b_{0}+b_{1} z+b_{2} z^{2}+\ldots+b_{n} z^{n}+\ldots
$$

Note that

$$
g\left(z^{2}\right)=\mathcal{G}(f)\left(z^{2}\right)=f(z) f(-z) .
$$

EXAMPLE. If $q$ is an odd integer and $f(z)=1-z^{q}$, we have $f(z) f(-z)=$ $\left(1-z^{q}\right)\left(1+z^{q}\right)=1-z^{2 q}$, and

$$
\mathcal{G}(f)=f .
$$

If $f$ is a polynomial of degree $n$ which does not vanish in 0 , and if $\widetilde{f}(z)=$ $z^{n} f(1 / z)$ is the reciprocal polynomial of $f$, then

$$
\mathcal{G}(\widetilde{f})=(-1)^{n} \widetilde{\mathcal{G}(f)} .
$$

It is obvious that, for any two series $f$ and $g$, we have the formulas

$$
\mathcal{G}(f g)=\mathcal{G}(f) \mathcal{G}(g)
$$

and, if $g(0)=1$,

$$
\mathcal{G}(f / g)=\mathcal{G}(f) / \mathcal{G}(g) .
$$

We shall often use the following notation for the iterates of $f$ under the transformation $\mathcal{G}$ :

$$
\begin{aligned}
& f_{0}=f, \quad f_{1}=\mathcal{G}(f), \\
& f_{2}=\mathcal{G}\left(f_{1}\right), \ldots, f_{k}=\mathcal{G}\left(f_{k-1}\right)=\mathcal{G}^{(k)}(f), \ldots
\end{aligned}
$$

Proposition 1. Let $f$ be a polynomial of degree $n$ with roots $z_{1}, z_{2}$, $\ldots, z_{n}$ and leading coefficient $a_{n}$. Then the polynomial $g=\mathcal{G}(f)$, where $\mathcal{G}$ is defined by (2.2), has leading coefficient $(-1)^{n} a_{n}^{2}$ and roots $z_{1}^{2}, \ldots, z_{n}^{2}$.

Proof. From the relations

$$
f(z)=a_{n}\left(z-z_{1}\right)\left(z-z_{2}\right) \ldots\left(z-z_{n}\right)
$$

and

$$
f(-z)=a_{n}\left(-z-z_{1}\right)\left(-z-z_{2}\right) \ldots\left(-z-z_{n}\right)
$$

it follows that

$$
f(z) f(-z)=(-1)^{n} a_{n}^{2}\left(z^{2}-z_{1}^{2}\right)\left(z^{2}-z_{2}^{2}\right) \ldots\left(z^{2}-z_{n}^{2}\right)
$$


and therefore, from (2.3),

$$
g(z)=\mathcal{G}(f)(z)=(-1)^{n} a_{n}^{2}\left(z-z_{1}^{2}\right)\left(z-z_{2}^{2}\right) \ldots\left(z-z_{n}^{2}\right) .
$$

In numerical analysis (cf. [4], [1] or [11]), the Graeffe method is used to compute an approximate value of the roots of a polynomial equation $f(x)=0$. The first step of the method is to calculate $f_{k}$ defined by (2.8) for $k$ large enough. From Proposition 1 , the roots of $f_{k}$ are $z_{1}^{2^{k}}, \ldots, z_{n}^{2^{k}}$, and, if we assume that $\left|z_{1}\right|>\ldots>\left|z_{n}\right|$, the sum of the roots of $f_{k}$ is close to $z_{1}^{2^{k}}$ which yields an approximate value for $\left|z_{1}\right|$. This old method is being revisited in computer algebra (cf. [3]).

Proposition 2. Let $f(z) \in \mathbb{C}[[z]], f(0) \neq 0$, and

$$
z \frac{f^{\prime}(z)}{f(z)}=\sum_{n=1}^{\infty} a_{n} z^{n}
$$

Then, for $k \geq 1$, we have

$$
\sum_{n=1}^{\infty} a_{2^{k} n} z^{n}=z \frac{f_{k}^{\prime}(z)}{f_{k}(z)}=\frac{z}{f_{k}(z)} \frac{d}{d z} f_{k}(z),
$$

where $f_{k}=\mathcal{G}^{(k)}(f)$ is defined by (2.2) and (2.8).

REMARK. Here and in what follows, $f_{k}^{\prime}$ will denote the derivative of $f_{k}$ (and not the $k$-iterate of $f^{\prime}$ ).

Proof of Proposition 2. We reason by induction on $k$. For $k=1$ and $z=y^{2}$, from $(2.10)$ and $(2.3)$ we have

$$
\begin{aligned}
\sum_{n=1}^{\infty} a_{2 n} z^{n} & =\sum_{n=1}^{\infty} a_{2 n} y^{2 n}=\frac{1}{2} \sum_{n=1}^{\infty}\left(a_{n} y^{n}+a_{n}(-y)^{n}\right) \\
& =\frac{1}{2}\left(y \frac{f^{\prime}(y)}{f(y)}-y \frac{f^{\prime}(-y)}{f(-y)}\right)=\frac{y}{2} \frac{f^{\prime}(y) f(-y)-f(y) f^{\prime}(-y)}{f(y) f(-y)} \\
& =\frac{y}{2 f_{1}\left(y^{2}\right)} \frac{d}{d y} f_{1}\left(y^{2}\right)=z \frac{f_{1}^{\prime}(z)}{f_{1}(z)}
\end{aligned}
$$

Further, the induction on $k$ is easy, by substituting $a_{2^{k} n}$ for $a_{2 n}$ and $f_{k-1}$ for $f$ in $(2.12)$.

Definition. We shall say that two power series $f, g$ with integral coefficients are congruent modulo $M$ (where $M$ is any positive integer) if their coefficients of the same degree are congruent modulo $M$. In other words, if

$$
f(z)=a_{0}+a_{1} z+a_{2} z^{2}+\ldots+a_{n} z^{n}+\ldots \in \mathbb{Z}[[z]]
$$

and

$$
g(z)=b_{0}+b_{1} z+b_{2} z^{2}+\ldots+b_{n} z^{n}+\ldots \in \mathbb{Z}[[z]]
$$


then

$$
f \equiv g(\bmod M) \Leftrightarrow \forall n \geq 0, a_{n} \equiv b_{n}(\bmod M) .
$$

Congruences of formal power series may be added or multiplied. If

$$
f \equiv g(\bmod M)
$$

and

$$
u \equiv v(\bmod M), \quad u \in \mathbb{Z}[[z]], v \in \mathbb{Z}[[z]]
$$

then

$$
f+u \equiv g+v(\bmod M) \quad \text { and } \quad f u \equiv g v(\bmod M) .
$$

One may differentiate (2.14) to get

$$
f^{\prime} \equiv g^{\prime}(\bmod M)
$$

Moreover, if $f(0)=g(0)=1,1 / f$ and $1 / g$ have integer coefficients and (2.14) holds, then

$$
\frac{1}{f} \equiv \frac{1}{g}(\bmod M)
$$

It is also easy to see that, for $f \in \mathbb{Z}[[z]]$ and $\mathcal{G}$ defined by (2.2), we have

$$
\mathcal{G}(f) \equiv f(\bmod 2) .
$$

Proposition 3. Let $f$ and $g$ be two formal power series with integral coefficients such that $f \equiv g(\bmod 2)$. Then, for $k \geq 0$, we have

$$
f_{k} \equiv g_{k}\left(\bmod 2^{k+1}\right) \text {, }
$$

where $f_{k}=\mathcal{G}^{(k)}(f)$ and $g_{k}=\mathcal{G}^{(k)}(g)$ are defined by (2.2) and (2.8).

Proof. Let us start by proving that if $u, v \in \mathbb{Z}[[z]]$ satisfy

$$
u \equiv v(\bmod 2 M)
$$

where $M$ is any positive integer, then $u_{1}=\mathcal{G}(u)$ and $v_{1}=\mathcal{G}(v)$ satisfy

$$
u_{1} \equiv v_{1}(\bmod 4 M) \text {. }
$$

It follows from $(2.20)$ that there exists $w \in \mathbb{Z}[[z]]$ such that

$$
u(z)=v(z)+2 M w(z) .
$$

Further, from (2.3),

$$
\begin{aligned}
u_{1}\left(z^{2}\right) & =u(z) u(-z)=(v(z)+2 M w(z))(v(-z)+2 M w(-z)) \\
& =v_{1}\left(z^{2}\right)+2 M[v(z) w(-z)+w(z) v(-z)]+4 M^{2} w_{1}\left(z^{2}\right),
\end{aligned}
$$

where $w_{1}=\mathcal{G}(w)$. But the expression in brackets is obviously congruent to 0 modulo 2 so that

$$
u_{1}\left(z^{2}\right) \equiv v_{1}\left(z^{2}\right)(\bmod 4 M),
$$

which, by substituting $z$ for $z^{2}$, yields (2.21). 
We prove Proposition 3 by induction on $k$. For $k=0$, from (2.8), (2.19) is just our hypothesis $f \equiv g(\bmod 2)$. Assume that $(2.19)$ holds for a nonnegative value of $k$; then applying (2.21) with $u=f_{k}, v=g_{k}$ and $M=2^{k}$ gives

$$
f_{k+1} \equiv g_{k+1}\left(\bmod 2^{k+2}\right)
$$

and the proof of Proposition 3 is complete.

3. Periodicity of $\sigma\left(\mathcal{A}, 2^{k} n\right) \bmod 2^{k+1}$. Let $\mathcal{B}$ be a finite set, and $N \geq$ $\max \mathcal{B}$ be an integer. For $i=0$ or $i=1$ we consider the set $\mathcal{A}=\mathcal{A}_{i}(\mathcal{B}, N)$ introduced in Section 1.

- If $i=0$, define the polynomial $P$ (already considered in [8]) by

$$
P(z)=\sum_{0 \leq n \leq J} \varepsilon_{n} z^{n}
$$

where $J$ is the largest integer such that $p(\mathcal{A}, J)$ is odd (such a $J$ does exist, since $p(\mathcal{A}, 0)=1$ ), and $\varepsilon_{n}$ is defined by

$$
p(\mathcal{A}, n) \equiv \varepsilon_{n}(\bmod 2), \quad \varepsilon_{n} \in\{0,1\} .
$$

It follows from (1.3) and (1.4) that

$$
F \equiv P(\bmod 2)
$$

- If $i=1$, we define $J$ as the smallest integer $\leq N+1$ such that $p(\mathcal{A}, n)$ is odd for all $n \geq J$ and $p(\mathcal{A}, J-1)$ is even. As observed in [8], such a $J \geq 2$ always exists, except in the case $\mathcal{B}=\{1\}$ which leads to

$$
\mathcal{A}_{1}(\{1\}, N)=\{1\} \quad \text { for all } N \geq 1 \text {. }
$$

The polynomial $P$ is now defined by $(3.1)$, with

$$
\varepsilon_{n}=\left\{\begin{array}{ll}
0 & \text { if } p(\mathcal{A}, n)-p(\mathcal{A}, n-1) \text { is even } \\
1 & \text { if } p(\mathcal{A}, n)-p(\mathcal{A}, n-1) \text { is odd }
\end{array} \quad(\text { for } n=0,1, \ldots, J)\right.
$$

with the convention (1.2). Note that the degree of $P$ is $J \leq N+1$. We have, from (1.3) and (1.4),

$$
F(z) \equiv \sum_{n=0}^{J-2} p(\mathcal{A}, n) z^{n}+\frac{z^{J}}{1-z} \equiv \frac{P(z)}{1-z}(\bmod 2) .
$$

Proposition 4. Except the case (3.4), the set $\mathcal{A}=\mathcal{A}_{i}(\mathcal{B}, N)$ defined by (1.5) and (1.4) is infinite.

Proof. If $\mathcal{A}=\mathcal{A}_{i}(\mathcal{B}, N)$ were finite, the product $\prod_{a \in \mathcal{A}}\left(1-z^{a}\right)$ would be a polynomial, say $Q(z)$, of degree $s=\sum_{a \in \mathcal{A}} a \geq \sum_{a \in \mathcal{B}} a$ and leading coefficient \pm 1 and, from (1.3), we should have

$$
F Q=1 \text {. }
$$


- If $i=0$, it would follow from (3.7), (3.3) and (2.15) that

$$
1 \equiv Q P(\bmod 2)
$$

which is impossible, since the leading term of $Q P$ has a positive degree, and its coefficient is \pm 1 .

- If $i=1,(3.7),(3.6)$ and (2.15) would yield

$$
1-z \equiv Q(z) P(z)(\bmod 2),
$$

which is also impossible if $s \geq 2$, i.e. $\mathcal{B} \neq\{1\}$.

Theorem 1. For any set $\mathcal{A}=\mathcal{A}_{i}(\mathcal{B}, N)$ (defined by (1.5) and (1.4)) and for any non-negative integer $k$, the sequence $\left(\sigma\left(\mathcal{A}, 2^{k} n\right)\right)_{n \geq 1}$ (where $\sigma$ is defined by (1.8)) satisfies a linear recurrence congruence modulo $2^{k+1}$, and therefore is periodic modulo $2^{k+1}$. Moreover, if $q_{k}$ denotes the smallest period, that is, the smallest positive integer $q_{k}$ such that

$$
\sigma\left(\mathcal{A}, 2^{k}\left(n+q_{k}\right)\right) \equiv \sigma\left(\mathcal{A}, 2^{k} n\right)\left(\bmod 2^{k+1}\right)
$$

for all $n \geq 1$, then, for all $k \geq 0$,

$$
q_{k} \text { divides } q_{k+1} \text {. }
$$

Proof. We start from the relation (1.9):

$$
z \frac{F^{\prime}(z)}{F(z)}=\sum_{n=1}^{\infty} \sigma(\mathcal{A}, n) z^{n}
$$

where $F(z)=F_{\mathcal{A}}(z)$ is defined by (1.3). By Proposition 2,

$$
\sum_{n=1}^{\infty} \sigma\left(\mathcal{A}, 2^{k} n\right) z^{n}=z \frac{F_{k}^{\prime}(z)}{F_{k}(z)}
$$

where $F_{k}$ is the $k$-iterate of $F$ under the transformation $\mathcal{G}$ (cf. (2.8)), and $F_{k}^{\prime}=(d / d z)\left(F_{k}(z)\right)$.

- Suppose that $i=0$. The congruence (3.3) holds with the polynomial $P$ defined by (3.1) and (3.2), and Proposition 3 implies that

$$
F_{k} \equiv P_{k}\left(\bmod 2^{k+1}\right)
$$

for all $k \geq 0$, with $P_{k}=\mathcal{G}^{(k)}(P)$. It follows from (1.2), (2.1), (3.1) and (3.2) that

$$
F_{k}(0)=P_{k}(0)=1
$$

and thus, from (2.15)-(2.17), (3.12) implies

$$
z \frac{F_{k}^{\prime}(z)}{F_{k}(z)} \equiv z \frac{P_{k}^{\prime}(z)}{P_{k}(z)}\left(\bmod 2^{k+1}\right) .
$$

Therefore, by (3.11) and (3.14),

$$
\sum_{n=1}^{\infty} \sigma\left(\mathcal{A}, 2^{k} n\right) z^{n} \equiv z \frac{P_{k}^{\prime}(z)}{P_{k}(z)}\left(\bmod 2^{k+1}\right) .
$$


But, for $k$ fixed, if $P_{k}(z)=a_{0}+a_{1} z+\ldots+a_{J} z^{J}$, then (3.15) implies that, for $n \geq J+1$

$$
\begin{aligned}
& a_{0} \sigma\left(\mathcal{A}, 2^{k} n\right) \\
& \quad \equiv-a_{1} \sigma\left(\mathcal{A}, 2^{k}(n-1)\right)-\ldots-a_{J} \sigma\left(\mathcal{A}, 2^{k}(n-J)\right)\left(\bmod 2^{k+1}\right) .
\end{aligned}
$$

It follows from (3.13) that $a_{0}=1$, so that (3.16) is a linear recurrence congruence, and, from a classical result based on the pigeonhole principle (cf. [2], for instance, for a detailed proof), it follows that $\sigma\left(\mathcal{A}, 2^{k} n\right) \bmod 2^{k+1}$ is periodic in $n$.

To show (3.9), observe first that a divisor of $2^{k+1} n$ is either a divisor of $2^{k} n$ or a multiple of $2^{k+1}$, and thus, from (1.8),

$$
\sigma\left(\mathcal{A}, 2^{k+1} n\right) \equiv \sigma\left(\mathcal{A}, 2^{k} n\right)\left(\bmod 2^{k+1}\right)
$$

But, from $(3.8), q_{k+1}$ is a period of $\sigma\left(\mathcal{A}, 2^{k+1} n\right) \bmod 2^{k+2}$, and thus, is also a period of $\sigma\left(\mathcal{A}, 2^{k+1} n\right) \bmod 2^{k+1}$ which is, by $(3.17)$, equal to $\sigma\left(\mathcal{A}, 2^{k} n\right) \bmod$ $2^{k+1}$ whose smallest period is $q_{k}$, and (3.9) is proved.

- Suppose now that $i=1$. The congruence $(3.6)$ will replace $(3.3) ;(3.11)$, (3.14) and (3.15) will become

$$
\sum_{n=1}^{\infty} \sigma\left(\mathcal{A}, 2^{k} n\right) z^{n}=z \frac{F_{k}^{\prime}(z)}{F_{k}(z)} \equiv z\left(\frac{P_{k}^{\prime}(z)}{P_{k}(z)}+\frac{1}{1-z}\right)\left(\bmod 2^{k+1}\right),
$$

and since the right hand side of (3.18) is a rational fraction, we conclude in the same way as in the case $i=0$.

LemMA 1. Let $Q(z) \in \mathbb{F}_{2}[z]$ be a polynomial of degree $d$ with $Q(0) \neq 0$. The order $\beta$ of $Q$ is the least positive integer such that $Q(z)$ divides $1+z^{\beta}$ in $\mathbb{F}_{2}[z]$. Then

(i) the positive integers $n$ such that $Q(z)$ divides $1+z^{n}$ in $\mathbb{F}_{2}[z]$ are the multiples of $\beta$;

(ii) the order of an irreducible polynomial of degree $d$ divides $2^{d}-1$ and thus is odd;

(iii) the order of a product of pairwise relatively prime polynomials is the lcm of the orders of the factors.

Proof. These are classical results in the theory of finite fields (cf. [5, Chap. 3, 3.6, 3.4 and 3.9].

LEMma 2. Let $m \geq 1$ be an integer and $Q_{1}, \ldots, Q_{m} \in \mathbb{F}_{2}[z]$ be coprime polynomials of positive degrees. Assume that there exists non-zero polynomials $A_{1}, \ldots, A_{m}$ satisfying $\left(A_{j}, Q_{j}\right)=1$ and $\operatorname{deg}\left(A_{j}\right)<\operatorname{deg}\left(Q_{j}\right)$ for $1 \leq j \leq m$ and

$$
\frac{A_{1}(z)}{Q_{1}(z)}+\ldots+\frac{A_{m}(z)}{Q_{m}(z)}=\frac{A(z)}{1+z^{T}} \quad \text { in } \mathbb{F}_{2}[z]
$$


where $T \geq 1$ is an integer and $A(z) \in \mathbb{F}_{2}[z]$. Then the order $\beta_{j}$ of $Q_{j}$ (cf. Lemma 1) satisfies

$$
\beta_{j} \text { divides } T, \quad 1 \leq j \leq m .
$$

Proof. Write $Q=Q_{1} \ldots Q_{m}, \widetilde{Q}_{j}=Q / Q_{j}, B=\sum_{j=1}^{m} A_{j} \widetilde{Q}_{j}$ so that

$$
\frac{A_{1}}{Q_{1}}+\ldots+\frac{A_{m}}{Q_{m}}=\frac{B}{Q}
$$

and

$$
B(z)\left(1+z^{T}\right)=A(z) Q(z)=A(z) Q_{1}(z) \ldots Q_{m}(z) .
$$

From our hypotheses, each $Q_{j}$ is coprime to $B$; therefore, $Q_{j}(z)$ divides $1+z^{T}$ and from Lemma $1(\mathrm{i}), \beta_{j}$ divides $T$.

Theorem 2. Let $P$ be the polynomial defined by (3.1) and (3.2) if $i=0$ and by (3.1) and (3.5) if $i=1$. Let the factorization of $P$ into irreducible factors over $\mathbb{F}_{2}[z]$ be

$$
P=Q_{1}^{\alpha_{1}} \ldots Q_{s}^{\alpha_{s}} .
$$

Denote by $d_{i}$ the degree of $Q_{i}$, by $\beta_{i}$ the order of $Q_{i}(z)$ (cf. Lemma 1$)$, and for all $k \geq 0$, set

$$
\begin{aligned}
& J_{k}=\left\{j: 1 \leq j \leq s, \alpha_{j} \equiv 2^{k}\left(\bmod 2^{k+1}\right)\right\}, \\
& I_{k}=J_{0} \cup \ldots \cup J_{k}=\left\{j: 1 \leq j \leq s, \alpha_{j} \not \equiv 0\left(\bmod 2^{k+1}\right)\right\}, \\
& T_{k}=\operatorname{lcm}_{j \in I_{k}} \beta_{j}
\end{aligned}
$$

(with $T_{k}=1$ if $I_{k}=\emptyset$ ). Then, for all $k \geq 0$, we have $q_{k}=T_{k}$, and $q_{k}$ is odd.

Note that if $2^{k_{0}}$ is the highest power of 2 dividing any exponent $\alpha_{j}$ in (3.19), then for $k>k_{0}$, we have $J_{k}=\emptyset, I_{k}=I_{k_{0}}$,

$$
q_{k}=q:=\operatorname{lcm}\left(\beta_{1}, \ldots, \beta_{s}\right)
$$

and moreover, from (3.9), $q$ is a common period for all the sequences $\left(\sigma\left(\mathcal{A}, 2^{k} n\right) \bmod 2^{k+1}\right)_{n \geq 1}, k \geq 0$.

Remark. Theorem 2 explains the examples given in [2] with $q_{0} \neq q_{1}$.

Proof of Theorem 2. In the whole proof, $k$ is a fixed non-negative integer.

- Assume $i=0$. To prove Theorem 2, we first consider polynomials $P$ and $Q_{j}$ as polynomials of $\mathbb{Z}[z]$ with coefficients 0 or 1 , so that (3.19) implies

$$
P \equiv Q_{1}^{\alpha_{1}} \ldots Q_{s}^{\alpha_{s}}(\bmod 2) .
$$

Then, it follows from (3.23), Proposition 3 and (2.6) that

$$
P_{k} \equiv\left(Q_{1}\right)_{k}^{\alpha_{1}} \ldots\left(Q_{s}\right)_{k}^{\alpha_{s}}\left(\bmod 2^{k+1}\right),
$$

where $P_{k}=\mathcal{G}^{(k)}(P)$ and $\left(Q_{j}\right)_{k}^{\alpha_{j}}=\left(\mathcal{G}^{(k)}\left(Q_{j}\right)\right)^{\alpha_{j}}$. By taking the logarithmic 
derivative of (3.24), we get (as in (3.14)) from (3.21)

$$
\frac{F_{k}^{\prime}}{F_{k}} \equiv \frac{P_{k}^{\prime}}{P_{k}} \equiv \sum_{j \in I_{k}} \alpha_{j} \frac{\left(Q_{j}\right)_{k}^{\prime}}{\left(Q_{j}\right)_{k}}\left(\bmod 2^{k+1}\right) .
$$

If we set

$$
V=\prod_{j \in I_{k}} Q_{j}
$$

then $V_{k}=\mathcal{G}^{(k)}(V)=\prod_{j \in I_{k}}\left(Q_{j}\right)_{k}$ is a common denominator for the right hand side of (3.25), and, if $S$ is the corresponding numerator, we have $\operatorname{deg} S<\operatorname{deg} V_{k}$ and $(3.25)$ reads

$$
\frac{P_{k}^{\prime}}{P_{k}} \equiv \frac{S}{V_{k}}\left(\bmod 2^{k+1}\right)
$$

Further, from Lemma 1 (iii) and (3.22), the order in $\mathbb{F}_{2}[z]$ of $V(z)$, defined by (3.26), is equal to $T_{k}$. So, there exists a polynomial $R \in \mathbb{Z}[z]$ such that

$$
V(z) R(z) \equiv 1-z^{T_{k}}(\bmod 2) .
$$

Now we consider $V$ as a polynomial of $\mathbb{Z}[z]$. By (2.6) and (2.4), Proposition 3 implies

$$
V_{k}(z) R_{k}(z) \equiv 1-z^{T_{k}}\left(\bmod 2^{k+1}\right)
$$

where $V_{k}=\mathcal{G}^{(k)}(V)$ and $R_{k}=\mathcal{G}^{(k)}(R)$. Then it follows from (3.15), (3.27) and (3.29) that

$$
\sum_{n=1}^{\infty} \sigma\left(\mathcal{A}, 2^{k} n\right) z^{n} \equiv z \frac{S(z) R_{k}(z)}{1-z^{T_{k}}}\left(\bmod 2^{k+1}\right) .
$$

Since $\operatorname{deg} S<\operatorname{deg} V_{k}$, the degree of $S R_{k}$ is smaller than $T_{k}$, and (3.30) shows that $\sigma\left(\mathcal{A}, 2^{k} n\right) \bmod 2^{k+1}$ is purely periodic with period $T_{k}$; therefore

$$
q_{k} \text { divides } T_{k} \text {. }
$$

Let us show now $q_{k}=T_{k}$ by induction. The definition of $q_{k}$ implies from (3.8) and (3.11) that for all $k \geq 0$,

$$
z \frac{F_{k}^{\prime}(z)}{F_{k}(z)} \equiv z \frac{W_{k}(z)}{1-z^{q_{k}}}\left(\bmod 2^{k+1}\right)
$$

where $W_{k}(z)=\sum_{n=1}^{q_{k}} \sigma\left(\mathcal{A}, 2^{k} n\right) z^{n-1}$.

For $k=0$, from $(3.25),(2.8),(3.20)$ and $(3.21)$, we have

$$
\frac{F^{\prime}}{F} \equiv \frac{P^{\prime}}{P} \equiv \sum_{j \in I_{0}} \alpha_{j} \frac{Q_{j}^{\prime}}{Q_{j}} \equiv \sum_{j \in J_{0}} \frac{Q_{j}^{\prime}}{Q_{j}}(\bmod 2) \text {. }
$$

If $I_{0}=J_{0}=\emptyset$, the above sum is empty and from $(3.10), \sigma(\mathcal{A}, n) \equiv 0$ $(\bmod 2)$ for all $n \geq 1$. Therefore, $q_{0}=T_{0}=1$. If $I_{0}=J_{0} \neq \emptyset$, from $(3.32)$ 
(with $k=0$ ) and (3.33) we deduce

$$
\sum_{j \in J_{0}} \frac{Q_{j}^{\prime}(z)}{Q_{j}(z)} \equiv \frac{W_{0}(z)}{1-z^{q_{0}}}(\bmod 2) .
$$

For each $j \in J_{0}$, it follows from Lemma 2 that $\beta_{j} \mid q_{0}$; thus, from (3.22), $T_{0} \mid q_{0}$, which, by (3.31), yields $q_{0}=T_{0}$.

Assume now that $k \geq 1$ and

$$
q_{l}=T_{l} \quad \text { for } 0 \leq l \leq k-1 .
$$

From (3.25) and (3.21) we have

$$
\frac{F_{k}^{\prime}(z)}{F_{k}(z)} \equiv \frac{P_{k}^{\prime}(z)}{P_{k}(z)} \equiv \sum_{j \in I_{k-1}} \alpha_{j} \frac{\left(Q_{j}^{\prime}\right)_{k}(z)}{\left(Q_{j}\right)_{k}(z)}+\sum_{j \in J_{k}} \alpha_{j} \frac{\left(Q_{j}^{\prime}\right)_{k}(z)}{\left(Q_{j}\right)_{k}(z)}\left(\bmod 2^{k+1}\right) .
$$

From our induction hypothesis (3.34) and from (3.22), for all $j \in I_{k-1}$, we have $\beta_{j} \mid q_{k-1}=T_{k-1}$; thus, from Lemma $1(\mathrm{i}), Q_{j}(z) \mid 1-z^{q_{k-1}}$ in $\mathbb{F}_{2}[z]$. Therefore, there exists a polynomial $Y_{j}(z) \in \mathbb{Z}[z]$ such that $1-z^{q_{k-1}} \equiv$ $Y_{j}(z) Q_{j}(z)(\bmod 2)$. From $(2.6),(2.4)$ and Proposition 3 , we have $1-z^{q_{k-1}} \equiv$ $\left(Y_{j}\right)_{k}(z)\left(Q_{j}\right)_{k}(z)\left(\bmod 2^{k+1}\right)$ so that we can write

$$
\sum_{j \in I_{k-1}} \alpha_{j} \frac{\left(Q_{j}^{\prime}\right)_{k}(z)}{\left(Q_{j}\right)_{k}(z)} \equiv \frac{B(z)}{1-z^{q_{k-1}}}\left(\bmod 2^{k+1}\right)
$$

where $B(z) \in \mathbb{Z}[z]$.

If $J_{k}=\emptyset$, it follows from (3.35), (3.36) and (3.11) that $q_{k-1}$ is a period of $\sigma\left(\mathcal{A}, 2^{k} n\right) \bmod 2^{k+1}$ so that $q_{k-1} \mid q_{k}$ which, by (3.9), implies $q_{k}=q_{k-1}$. Since $I_{k}=I_{k-1}$, from (3.21) and (3.34) we have $T_{k}=T_{k-1}=q_{k-1}=q_{k}$.

If $J_{k} \neq \emptyset,(3.35)$ can be rewritten, by (3.36), (3.32) and (3.9), as

$$
\begin{aligned}
\sum_{j \in J_{k}} \alpha_{j} \frac{\left(Q_{j}^{\prime}\right)_{k}(z)}{\left(Q_{j}\right)_{k}(z)} & \equiv \frac{F_{k}^{\prime}(z)}{F_{k}(z)}-\sum_{j \in I_{k-1}} \alpha_{j} \frac{\left(Q_{j}^{\prime}\right)_{k}(z)}{\left(Q_{j}\right)_{k}(z)} \\
& \equiv \frac{W_{k}(z)}{1-z^{q_{k}}}-\frac{B(z)}{1-z^{q_{k-1}}} \equiv \frac{B_{1}(z)}{1-z^{q_{k}}}\left(\bmod 2^{k+1}\right)
\end{aligned}
$$

where $B_{1}(z) \in \mathbb{Z}[z]$ is a polynomial of degree less than $q_{k}$. In (3.37), from (3.20), the $\alpha_{j}$ 's are multiples of $2^{k}$, so are also the coefficients of $B_{1}$, and (3.37) implies

$$
\sum_{j \in J_{k}} \frac{\alpha_{j}}{2^{k}} \cdot \frac{\left(Q_{j}^{\prime}\right)_{k}(z)}{\left(Q_{j}\right)_{k}(z)} \equiv \frac{B_{1}(z) / 2^{k}}{1-z^{q_{k}}}(\bmod 2) .
$$

From (3.20), $\alpha_{j} / 2^{k}$ is odd, and from (2.18), (2.17) and (2.15), we get

$$
\sum_{j \in J_{k}} \frac{Q_{j}^{\prime}(z)}{Q_{j}(z)} \equiv \frac{B_{1}(z) / 2^{k}}{1-z^{q_{k}}}(\bmod 2) \text {. }
$$


By Lemma 2, this implies that, for $j \in J_{k}$, we have $\beta_{j} \mid q_{k}$ so that $T_{k} \mid q_{k}$, which, together with (3.31), yields $q_{k}=T_{k}$.

The oddness of $q_{k}=T_{k}$ results from Lemma 1(ii) and (3.22), and the proof of Theorem 2 is complete when $i=0$.

- Assume $i=1$. From (3.18), (3.25) becomes

$$
\frac{F_{k}^{\prime}(z)}{F_{k}(z)} \equiv \frac{P_{k}^{\prime}(z)}{P_{k}(z)}+\frac{1}{1-z} \equiv \frac{1}{1-z}+\sum_{j \in I_{k}} \alpha_{j} \frac{\left(Q_{j}\right)_{k}^{\prime}}{\left(Q_{j}\right)_{k}}\left(\bmod 2^{k+1}\right) .
$$

The polynomial $1-z^{T_{k}}$, where $T_{k}$ is defined by (3.22), is still a common denominator for the right hand side of (3.38), and (3.31) can be proved in the same way as in the case $i=0$. The proof of $q_{k}=T_{k}$ follows by replacing (3.25) by (3.38).

4. Relations between $\mathcal{A}_{1}(\mathcal{B}, N)$ and $\mathcal{A}_{0}\left(\mathcal{B}^{\prime}, N^{\prime}\right)$. In this section, we want to show that the sets $\mathcal{A}_{1}(\mathcal{B}, N)$ do not differ very much of the sets $\mathcal{A}_{0}(\mathcal{B}, N)$. More precisely, by adding or subtracting powers of 2 to $\mathcal{A}_{1}(\mathcal{B}, N)$, one can get a set $\mathcal{A}_{0}\left(\mathcal{B}^{\prime}, N+1\right)$ for a suitable set $\mathcal{B}^{\prime} \subset\{1, \ldots, N+1\}$.

Theorem 3. Let $\mathcal{A}=\mathcal{A}_{1}(\mathcal{B}, N)$ be defined by (1.5) and (1.4) with $\mathcal{B}$ any set different from $\{1\}$, and $N$ any integer satisfying $N \geq \max \mathcal{B}$.

(i) Denote by $2^{h}, h \geq 0$, the smallest element (if it exists) of $\mathcal{A}$ which is a power of 2 . Then

$$
\mathcal{A}^{\prime}=\mathcal{A} \cup\left\{1,2, \ldots, 2^{h-1}\right\} \backslash\left\{2^{h}\right\}=\mathcal{A}_{0}\left(\mathcal{B}^{\prime}, N+1\right)
$$

with

(ii) If $\mathcal{A} \cap\left\{1,2, \ldots, 2^{h}, \ldots\right\}=\emptyset$, then

$$
\mathcal{A}^{\prime}=\mathcal{A} \cup\left\{1,2, \ldots, 2^{h}, \ldots\right\}=\mathcal{A}_{0}\left(\mathcal{B}^{\prime}, N+1\right)
$$

with $\mathcal{B}^{\prime}$ still defined by (4.2).

Proof. (i) From (1.3), we have

$$
\begin{aligned}
\sum_{n=0}^{\infty} p\left(\mathcal{A}^{\prime}, n\right) z^{n} & =\prod_{a \in \mathcal{A}^{\prime}} \frac{1}{1-z^{a}}=\frac{1-z^{2^{h}}}{(1-z) \ldots\left(1-z^{2^{h-1}}\right)} \prod_{a \in \mathcal{A}} \frac{1}{1-z^{a}} \\
& \equiv \frac{1-z^{2^{h}}}{(1+z) \ldots\left(1+z^{2^{h-1}}\right)} \prod_{a \in \mathcal{A}} \frac{1}{1-z^{a}}(\bmod 2) \\
& \equiv(1-z) \prod_{a \in \mathcal{A}} \frac{1}{1-z^{a}} \equiv(1-z) \sum_{n=0}^{\infty} p(\mathcal{A}, n) z^{n}(\bmod 2) .
\end{aligned}
$$

Hence

$$
p\left(\mathcal{A}^{\prime}, n\right) \equiv p(\mathcal{A}, n)-p(\mathcal{A}, n-1)(\bmod 2),
$$


so that, from (1.4), $p\left(\mathcal{A}^{\prime}, n\right)$ is even for $n \geq N+2$; therefore, (4.1) follows from (1.6).

(ii) The argument is similar, by observing that

$$
(1+z)\left(1+z^{2}\right) \ldots\left(1+z^{2^{h}}\right) \ldots=\frac{1}{1-z} .
$$

\section{References}

[1] E. H. Bareiss, Resultant procedure and the mechanisation of the Graeffe process, J. Assoc. Comput. Mach. 7 (1960), 346-386.

[2] F. Ben saïd, On a conjecture of Nicolas-Sárközy about partitions, J. Number Theory 95 (2002), 209-226.

[3] X. Gourdon, thèse, http://pauillac.inria.fr/algo/gourdon/thesis/html

[4] C. H. Graeffe, Die Auflösung der höheren numerischen Gleichungen, Zurich, 1837.

[5] R. Lidl and H. Niederreiter, Introduction to Finite Fields and their Applications, Cambridge Univ. Press, revised edition, 1994.

[6] J.-L. Nicolas, On the parity of generalised partition functions II, Period. Math. Hungar. 43 (2001), 177-189.

[7] J.-L. Nicolas, I. Z. Ruzsa and A. Sárközy, On the parity of additive representation functions, J. Number Theory 73 (1998), 292-317, with an appendix in French by J.-P. Serre.

[8] J.-L. Nicolas and A. Sárközy, On the parity of generalized partition functions, in: Proc. Millennium Conference (Urbana, IL, 2000), to appear.

[9] K. Ono, Parity of the partition function in arithmetic progressions, J. Reine Angew. Math. 472 (1996), 1-15.

[10] - Distribution of the partition function modulo m, Ann. of Math. 151 (2000), 293-307.

[11] A. Ralston and P. Rabinowitz, A First Course in Numerical Analysis, McGraw-Hill, 1978.

Faculté des Sciences de Monastir

Institut Girard Desargues Avenue de l'environnement

UMR 5028

5000, Monastir, Tunisie

E-mail: Fethi.BenSaid@fsm.rnu.tn

Bât. Doyen Jean Braconnier Université Claude Bernard (Lyon 1)

21 Avenue Claude Bernard F-69622 Villeurbanne Cedex, France E-mail: jlnicola@in2p3.fr

Received on 23.10.2001

and in revised form on 7.3.2002 